\title{
Broadcasting with a Battery Limited Energy Harvesting Rechargeable Transmitter
}

\author{
Omur Ozel $^{1}$, Jing Yang ${ }^{2}$, and Sennur Ulukus ${ }^{1}$ \\ ${ }^{1}$ Department of Electrical and Computer Engineering, University of Maryland, College Park, MD 20742 \\ ${ }^{2}$ Department of Electrical and Computer Engineering, University of Wisconsin-Madison, Madison, WI 53706
}

\begin{abstract}
We consider the minimization of the transmission completion time with a battery limited energy harvesting transmitter in a two-user AWGN broadcast channel. The transmitter has fixed number of packets for each receiver and energy is modeled to arrive (be harvested) at the transmitter at random instants. The battery at the transmitter has a finite storage capacity, hence energy may overflow without being utilized for data transmission. We derive the optimal offline transmission policy that minimizes the time by which all of the data packets are delivered to their respective destinations. We analyze the structural properties of the optimal transmission policy using a dual problem. We find the optimal total transmit power sequence by a directional water-filling algorithm. We prove that there exists a cut-off power level such that if the allocated power is lower than this level, then only the stronger user is served in that epoch; otherwise, the power above this level is allocated to the weaker user. Based on these properties, we propose an algorithm that gives the globally optimal offline policy. The proposed algorithm uses directional water-filling repetitively.
\end{abstract}

\section{INTRODUCTION}

Energy harvesting communication systems have been widely used in many wireless networking applications as they bring improved lifetime and ease of deployment. A distinctive characteristic of these systems is that energy becomes available for use in communication during the course of transmission of data. This requires the adaptation of the transmission policies to the energy arrivals. In this paper, we consider data transmission with an energy harvesting transmitter in a broadcast setting. We build upon our previous work [1] and derive the optimal offline policy that achieves the minimum transmission completion time when the transmitter has a finite capacity battery.

As shown in Fig. 1, we consider a broadcast channel with an energy harvesting transmitter and two receivers. Three queues at the transmitter are: two data queues that store the data destined to the two receivers and an energy queue (battery) that stores the harvested energy. The energy queue has a finite capacity and can store at most $E_{\max }$ units of energy. As shown in Fig. 2, the energy arrives (is harvested) at times $s_{k}$ in the amounts $E_{k} . E_{0}$ is the initial energy available in the battery at time zero. Saving energy for future use is advantageous, however, finite battery capacity constrains this capability, and thus necessitates avoiding energy overflows [2], [3]. We focus on the optimal offline policy that minimizes the time, $T$,

This work was supported by NSF Grants CCF 07-29127, CNS 09-64632, CCF 09-64645, CCF 10-18185.

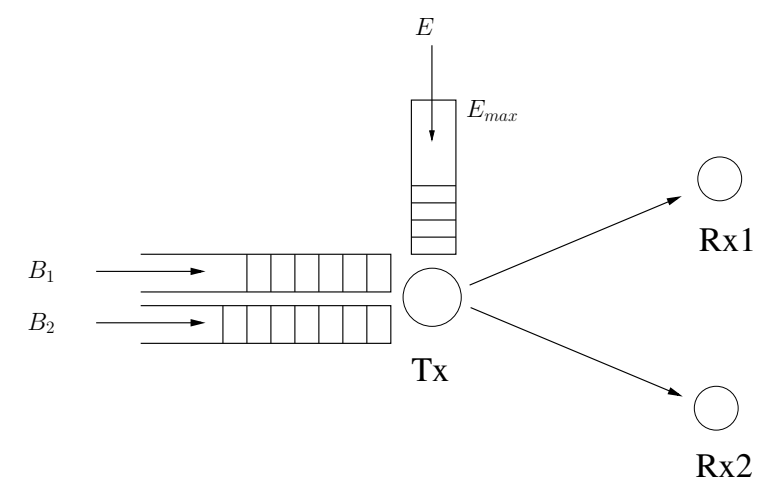

Fig. 1. Broadcasting $\left(B_{1}, B_{2}\right)$ bits with an energy harvesting transmitter with a finite capacity battery.

required to transmit $B_{1}$ bits to receiver 1 and $B_{2}$ bits to receiver 2 . The transmission policy is subject to the causality of energy arrivals as well as the finite battery constraint.

Data transmission in energy harvesting systems has attracted recent attention [1]-[6]. Transmission completion time minimization problem in a point-to-point communication channel is solved in [4], [5] without battery constraints, and later in [2] with finite battery capacity constraints. In [3], we extend the analysis to the fading channel through a concise algorithm called directional water-filling. In [1], we solve the transmission completion time minimization problem in a twouser broadcast channel, independently and concurrently with [6]. Both works assume that the transmitter battery size is unlimited. This paper extends these works to the case of a transmitter with a finite capacity battery.

In [1], we show, under the assumption of an infinite sized battery, that the time sequence of total power increases monotonically as in the single user case in [5]. Moreover, in [1], we prove that there exists a cut-off power level for the power shares of the strong and weak users; strong user's power share is always greater than or equal to this cut-off level. The structure of the optimal policy in [1] is contingent upon the availability of an infinite capacity battery. In particular, when a large amount of energy is harvested, the development in [1] assumes that some portion of this harvested energy can be saved for future use. However, when the battery capacity is finite, energy may overflow in such cases. Therefore, the added challenge in the finite capacity battery case is to accommodate 


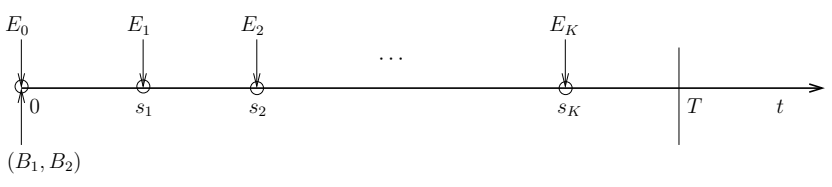

Fig. 2. Energies arrive at time instants $s_{k}$ in amounts $E_{k}$.

every bit of the incoming energy by carefully managing the transmission power and users' power shares according to the times and amounts of energy arrivals.

Interestingly, we find, in the current paper, that as in [1] the determination of the total transmit power can be separated from the determination of the shares of receiver 1 and 2 without losing optimality. We reach this result by following our approach in [1]. We, first, obtain the structural properties of the optimal policy by means of a dual problem, namely, the maximization of the weighted sum of bits served for receivers 1 and 2 by a deadline $T$. In particular, we show that the total power in each epoch must be the same as the total power in the single-user channel, which can be found by a directional water-filling algorithm in [3]. Then, we find the optimal policy in our broadcast scenario by an iterative algorithm that makes repetitive use of directional water-filling.

\section{System Model and Problem Formulation}

The system model is as shown in Fig. 1 . There are $\left(B_{1}, B_{2}\right)$ bits destined for receivers 1 and 2. $E_{0}$ is the initial energy available in the battery at time zero and energy arrivals occur at times $\left\{s_{1}, s_{2}, \ldots\right\}$ in amounts $\left\{E_{1}, E_{2}, \ldots\right\}$. We call the time interval between two energy arrivals an epoch. The epoch lengths are $\ell_{i}=s_{i}-s_{i-1}$ with the convention $s_{0}=0$. A standing assumption in the paper is $E_{i} \leq E_{\max }$ for all $i$, as otherwise the excess energy $E_{i}-E_{\max }$ cannot be stored in the battery anyway.

We model the physical layer as an AWGN broadcast channel, where the received signals at the two receivers are

$$
\begin{aligned}
& Y_{1}=X+Z_{1} \\
& Y_{2}=X+Z_{2}
\end{aligned}
$$

where $X$ is the transmit signal, and $Z_{1}$ and $Z_{2}$ are Gaussian noises with zero-mean and variances 1 and $\sigma^{2}$, respectively, where $\sigma^{2}>1$. Therefore, the second user is the degraded (weaker) user in our broadcast channel. Assuming that the transmitter transmits with power $P$, the capacity region for this two-user AWGN broadcast channel is [7]

$$
\begin{aligned}
& r_{1} \leq \frac{1}{2} \log _{2}(1+\alpha P) \\
& r_{2} \leq \frac{1}{2} \log _{2}\left(1+\frac{(1-\alpha) P}{\alpha P+\sigma^{2}}\right)
\end{aligned}
$$

where $\alpha$ is the fraction of power spent for the message transmitted to the first user. This capacity region is shown in Fig. 3.

Let us denote $f(p) \triangleq \frac{1}{2} \log _{2}(1+p)$ for future use. After necessary rearrangements, we can show that the rate pairs

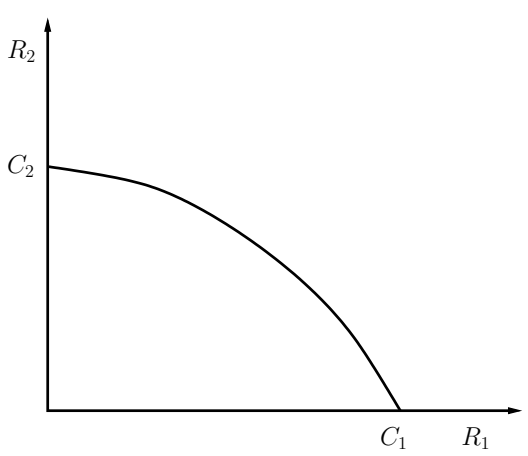

Fig. 3. The capacity region of the two-user AWGN broadcast channel.

$\left(r_{1}, r_{2}\right)$ on the boundary of the capacity region satisfy

$$
\begin{aligned}
P & =2^{2\left(r_{1}+r_{2}\right)}+\left(\sigma^{2}-1\right) 2^{2 r_{2}}-\sigma^{2} \\
& \triangleq g\left(r_{1}, r_{2}\right)
\end{aligned}
$$

Let us denote the transmit power at time $t$ as $P(t)$ for $t \in[0, T]$. Then, the total energy consumed by the transmitter up to time $t$ can be expressed as $\int_{0}^{t} P(\tau) d \tau$. Note that because of the finite battery capacity constraint, at any time $t$, if the unconsumed energy is greater than $E_{\max }$, only $E_{\max }$ can be stored in the battery and the rest of the energy overflows and hence is wasted. This may happen only at the instants of energy arrival. Therefore, the total removed energy from the battery at $s_{k}$, including the consumed part and the wasted part, can be expressed recursively as

$$
\begin{aligned}
E_{r}\left(s_{k}\right)=\max \left\{E_{r}\left(s_{k-1}\right)+\int_{s_{k-1}}^{s_{k}} P(\tau) d \tau,\right. & \\
& \left.\left(\sum_{j=0}^{k} E_{j}-E_{\text {max }}\right)^{+}\right\}, \quad k=1,2, \ldots
\end{aligned}
$$

where $(x)^{+}=\max \{0, x\}$, the upper limit of the integral $s_{k}$ should be interpreted as $s_{k}-\epsilon$ for small $\epsilon>0$, and $E_{r}\left(s_{0}\right)=$ 0 . We can extend the definition of $E_{r}$ for the times $t \neq s_{k}$ as:

$$
E_{r}(t)=E_{r}\left(s_{h(t)}\right)+\int_{s_{h(t)}}^{t} P(\tau) d \tau
$$

where $h(t)=\max \left\{i: s_{i}<t\right\}$. An illustration of $E_{r}(t)$ is shown in Fig. 4. The upper curve in Fig. 4 represents the total energy arrived and the lower curve is obtained by subtracting $E_{\max }$ from the upper curve. $E_{r}(t)$ always lies in between these two curves. In the particular $E_{r}(t)$ shown in Fig. 4, the energy in the battery exceeds $E_{\max }$ at the time of third energy arrival at $s_{3}$ and some energy is removed from the battery without being utilized for data transmission. After $s_{3}$, energy removal from the battery continues due to the data transmission and hence the removal curve approaches the total energy arrival curve indicating that the battery energy is decreasing. In general, battery energy is decreasing in $\left(s_{i-1}, s_{i}\right)$ for all $i$.

As the transmitter cannot utilize the energy that has not yet 


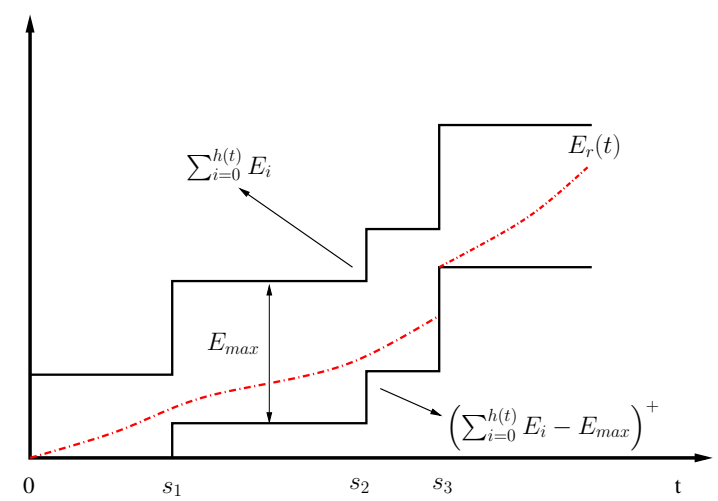

Fig. 4. The total removed energy curve $E_{r}(t)$. The jump at $s_{3}$ represents an energy overflow because of the finite battery capacity limit.

arrived, the transmission policy is subject to an energy causality constraint. The removed energy $E_{r}(t)$ cannot exceed the total energy arrival during the communication. This constraint is mathematically stated as follows:

$$
E_{r}(t) \leq \sum_{i=0}^{h(t)} E_{i}, \quad \forall t \in[0, T]
$$

As the energies arrive at discrete times, the causality constraint reduces to inequalities that has to be satisfied at the times of energy arrivals:

$$
E_{r}\left(s_{k-1}\right)+\int_{s_{k-1}}^{s_{k}} P(\tau) d \tau \leq \sum_{i=0}^{k-1} E_{i}, \quad \forall k
$$

A transmission policy guarantees no-energy-overflow if the following constraint is satisfied:

$$
\sum_{i=0}^{h(t)} E_{i}-\int_{0}^{t} P(\tau) d \tau \leq E_{\max }, \quad \forall t \in[0, T]
$$

The constraint in (11) imposes that at least $\sum_{i=0}^{k} E_{i}-E_{\max }$ amount of energy has been consumed by the time the $k$ th energy arrives so that the battery can accommodate $E_{k}$ at time $s_{k}$. If a policy satisfies (11), the causality constraint in (10) can be simplified to the following as depicted in Fig. 5:

$$
\int_{0}^{s_{k}} P(\tau) d \tau \leq \sum_{i=0}^{k-1} E_{i}, \quad \forall k
$$

In Fig. 5, the total energy curve of the policy does not intersect the lower curve at the vertically rising parts and thus no energy is removed from the battery due to energy overflows. Hence, the causality constraint reduces to the condition that the total energy curve must lie below the upper curve in Fig. 5.

The transmission policy in a broadcast channel is comprised of the total power $P(t)$ and the portions of the total transmit power $\alpha(t)$ and $1-\alpha(t)$. However, it can be identically represented by rates $r_{1}(t)$ and $r_{2}(t)$ via (3)-(4). We call any rate policy $\left(r_{1}(t), r_{2}(t)\right)$ feasible if it satisfies the causality constraint in (12) where $P(t)=g\left(r_{1}(t), r_{2}(t)\right)$ is the power

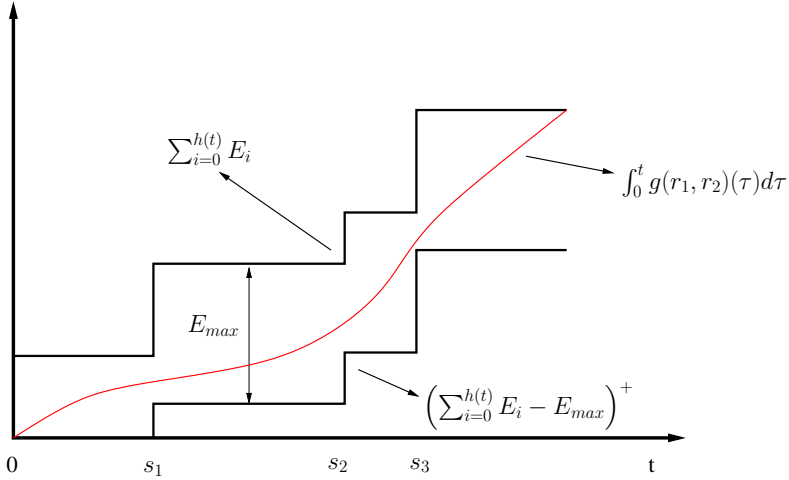

Fig. 5. Energy causality constraint and no-energy-overflow constraint are depicted as cumulative energy curves and the power consumption curve of a transmission policy that simultaneously satisfies these two constraints by lying in between these two curves.

necessary to achieve $\left(r_{1}(t), r_{2}(t)\right)$ pair.

Instead of directly finding the optimal policy that minimizes the transmission completion time, we first identify the maximum departure region of bits delivered to both users by any fixed time $T$ in the next section.

\section{The Dual Problem in the Two-User Broadcast ChANNEL}

In this section, we consider the dual problem which is to determine the maximum departure region [1] which gives the set of number of bits that can be delivered to the two receivers by a fixed deadline $T$. Let $K$ denote the number of energy arrivals in $[0, T)$ yielding $K+1$ epochs and let us set $s_{K+1}=T$. Let $\left(B_{1}, B_{2}\right)$ be the total number of bits sent to receivers 1 and 2 , respectively, over the duration $[0, T]$, i.e., $B_{1}=\int_{0}^{T} r_{1}(\tau) d \tau$ and $B_{2}=\int_{0}^{T} r_{2}(\tau) d \tau$. We define the maximum departure region as follows.

Definition 1 For any fixed transmission duration $T$, the maximum departure region, denoted as $\mathcal{D}(T)$, is the union of $\mathcal{R}\left(B_{1}, B_{2}\right)=\left\{\left(b_{1}, b_{2}\right): 0 \leq b_{1} \leq B_{1} ; 0 \leq b_{2} \leq B_{2}\right\}$ where $\left(B_{1}, B_{2}\right)$ is the total number of bits sent by some rate allocation policy that satisfies the energy causality and noenergy-overflow conditions. That is,

$$
\mathcal{D}(T)=\bigcup_{\left(r_{1}(t), r_{2}(t)\right)} \mathcal{R}\left(B_{1}, B_{2}\right)
$$

where $\left(r_{1}(t), r_{2}(t)\right)$ satisfies the causality constraint

$$
\int_{0}^{t} g\left(r_{1}, r_{2}\right)(\tau) d \tau \leq \sum_{i=0}^{h(t)} E_{i}, \quad \forall t \in[0, T)
$$

and the no-energy-overflow constraint

$$
\sum_{i=0}^{h(t)} E_{i}-\int_{0}^{t} g\left(r_{1}, r_{2}\right)(\tau) d \tau \leq E_{\max }, \quad \forall t \in[0, T)
$$

The departure region of any policy that causes energy overflow can be dominated by a policy that does not allow energy 
overflows. Hence, in the definition of $\mathcal{D}(T)$, we restrict the policies to satisfy the no-energy-overflow condition in (11).

We call any policy that achieves the boundary of $\mathcal{D}(T)$ to be optimal. The transmission rates remain constant between energy harvests under any optimal policy (c.f. Lemma 1 in [1]). Therefore, in the sequel, we restrict ourselves to policies in which the rates remain constant between any two consecutive energy arrivals. We denote the rates that go to users as $\left(r_{1 i}, r_{2 i}\right)$ over the duration $\left[s_{i-1}, s_{i}\right)$. The causality constraint in (12) reduces to the following constraint on $\left(r_{1 i}, r_{2 i}\right)$ :

$$
\sum_{i=1}^{k} g\left(r_{1 i}, r_{2 i}\right) \ell_{i} \leq \sum_{i=0}^{k-1} E_{i}, \quad k=1, \ldots, K+1
$$

and the no-energy-overflow condition in (11) reduces to:

$$
\sum_{i=0}^{k} E_{i}-\sum_{i=1}^{k} g\left(r_{1 i}, r_{2 i}\right) \ell_{i} \leq E_{\max }, \quad k=1, \ldots, K
$$

An important property of $\mathcal{D}(T)$ is stated next.

\section{Lemma $1 \mathcal{D}(T)$ is a convex region.}

Proof: Assume that $\left(B_{1}, B_{2}\right)$ and $\left(B_{1}^{\prime}, B_{2}^{\prime}\right)$ are two points that can be achieved by some policies $\left\{\left(r_{1 i}, r_{2 i}\right)\right\}_{i=1}^{K+1}$ and $\left\{\left(r_{1 i}^{\prime}, r_{2 i}^{\prime}\right)\right\}_{i=1}^{K+1}$, respectively, that satisfy the energy causality constraint in (16) and the no-energy-overflow constraint in (17) such that

$$
\begin{aligned}
& \left(B_{1}, B_{2}\right)=\left(\sum_{i=1}^{K+1} r_{1 i} \ell_{i}, \sum_{i=1}^{K+1} r_{2 i} \ell_{i}\right) \\
& \left(B_{1}^{\prime}, B_{2}^{\prime}\right)=\left(\sum_{i=1}^{K+1} r_{1 i}^{\prime} \ell_{i}, \sum_{i=1}^{K+1} r_{2 i}^{\prime} \ell_{i}\right)
\end{aligned}
$$

We will show that there exists a policy that achieves $\left(\lambda B_{1}+\right.$ $\left.\bar{\lambda} B_{1}^{\prime}, \lambda B_{2}+\bar{\lambda} B_{2}^{\prime}\right)$ where $\bar{\lambda}=1-\lambda$.

By the convexity of $g\left(r_{1}, r_{2}\right)$ in (6), we have

$$
g\left(\lambda r_{1}+\bar{\lambda} r_{1}^{\prime}, \lambda r_{2}+\bar{\lambda} r_{2}^{\prime}\right) \leq \lambda g\left(r_{1}, r_{2}\right)+\bar{\lambda} g\left(r_{1}^{\prime}, r_{2}^{\prime}\right)
$$

Hence, transmission of convex combination of $\left(B_{1}, B_{2}\right)$ and $\left(B_{1}^{\prime}, B_{2}^{\prime}\right)$ requires less energy than the convex combination of the energies required to transmit them separately. Therefore, the rate allocation $\left\{\lambda r_{1 i}+\bar{\lambda} r_{1 i}^{\prime}, \lambda r_{2 i}+\bar{\lambda} r_{2 i}^{\prime}\right\}_{i=1}^{K+1}$ may not satisfy the no-energy-overflow constraints in (17), though, it achieves $\left(\lambda B_{1}+\bar{\lambda} B_{1}^{\prime}, \lambda B_{2}+\bar{\lambda} B_{2}^{\prime}\right)$ in the $[0, T]$ interval. if this is the case, we can always increase the energy consumption so that we get a new policy that achieves the desired point while satisfying the no-energy-overflow and causality constraints. Let us define the new policy $\left\{\left(r_{1 i}^{\prime \prime}, r_{2 i}^{\prime \prime}\right)\right\}$ as $r_{1 i}^{\prime \prime} \geq \lambda r_{1 i}+\bar{\lambda} r_{1 i}^{\prime}$ and $r_{2 i}^{\prime \prime} \geq \lambda r_{2 i}+\bar{\lambda} r_{2 i}^{\prime}$ for all $i$ so that we have

$$
g\left(r_{1 i}^{\prime \prime}, r_{2 i}^{\prime \prime}\right)=\lambda g\left(r_{1 i}, r_{2 i}\right)+\bar{\lambda} g\left(r_{1 i}^{\prime}, r_{2 i}^{\prime}\right)
$$

Since $g\left(r_{1}, r_{2}\right)$ is strictly monotone and continuous in $r_{1}, r_{2}$, one can always find $\left\{r_{1 i}^{\prime \prime}, r_{2 i}^{\prime \prime}\right\}_{i=1}^{K+1}$ as desired.

Clearly $\left\{\left(r_{1 i}^{\prime \prime}, r_{2 i}^{\prime \prime}\right)\right\}$ satisfies the energy causality and noenergy-overflow conditions and the resulting operating point

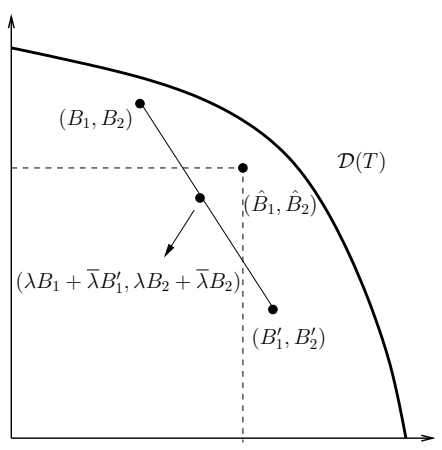

Fig. 6. The maximum departure region $\mathcal{D}(T)$ is a convex region.

$\left(\hat{B}_{1}, \hat{B}_{2}\right)$ is such that

$$
\begin{aligned}
& \hat{B}_{1} \geq \lambda B_{1}+\bar{\lambda} B_{1}^{\prime} \\
& \hat{B}_{2} \geq \lambda B_{2}+\bar{\lambda} B_{2}^{\prime}
\end{aligned}
$$

Hence, any convex combination of $\left(B_{1}, B_{2}\right)$ and $\left(B_{1}^{\prime}, B_{2}^{\prime}\right)$ can be achieved by some policy that obeys energy causality and no-energy-overflow constraints. This proves the convexity of $\mathcal{D}(T)$. We illustrate the main steps of this proof in Fig. 6 .

Since $\mathcal{D}(T)$ is a convex region (in fact, it is strictly convex) its boundary can be characterized by solving the following optimization problem for all $\mu_{1}, \mu_{2} \geq 0$,

$$
\begin{aligned}
\max _{\mathbf{r}_{1}, \mathbf{r}_{2}} & \mu_{1} \sum_{i=1}^{K+1} r_{1 i} \ell_{i}+\mu_{2} \sum_{i=1}^{K+1} r_{2 i} \ell_{i} \\
\text { s.t. } & \sum_{i=1}^{k} g\left(r_{1 i}, r_{2 i}\right) \ell_{i} \leq \sum_{i=0}^{k-1} E_{i}, 1 \leq k \leq K+1 \\
& \sum_{i=0}^{k} E_{i}-\sum_{i=1}^{k} g\left(r_{1 i}, r_{2 i}\right) \ell_{i} \leq E_{\text {max }}, 1 \leq k \leq K
\end{aligned}
$$

where $K$ is the number of energy arrivals in $[0, T)$, and $K+1$ is the number of epochs. Here, $\mathbf{r}_{1}$ and $\mathbf{r}_{2}$ denote the time sequence of rates $r_{1 i}$ and $r_{2 i}$, for $i=1, \ldots, K+1$ that go to users 1 and 2, respectively.

The problem in (24) is a constrained maximization problem with a linear objective function. However, the constraint set is non-convex because of the direction of the inequality in the no-energy-overflow condition. The underlying reason for this is that, even if the two policies do not cause energy overflows themselves, their convex combination may cause energy overflows. Therefore, in principle, there may exist multiple extrema for the problem in (24). Next, we argue that in fact there exists a unique global optimum solution for this problem. To that end, we extend the set of possible rate policies to include those that allow energy overflows. Let us denote the extended set of feasible policies that allow energy overflows by $\mathcal{P}_{R}(T)$ :

$$
\mathcal{P}_{R}(T) \triangleq\left\{\left\{\left(r_{1 i}, r_{2 i}\right)\right\}_{i=1}^{K+1}: E_{r}\left(s_{k}\right) \leq \sum_{i=0}^{k-1} E_{i}, \forall k\right\}
$$


Then, we have the following result.

Lemma $2 \mathcal{P}_{R}(T)$ is a convex set.

Proof: Let two policies $\left\{\left(r_{1 i}, r_{2 i}\right)\right\}_{i=1}^{K+1},\left\{\left(r_{1 i}^{\prime}, r_{2 i}^{\prime}\right)\right\}_{i=1}^{K+1}$ be in $\mathcal{P}_{R}(T)$ and let their energy removal curves be $E_{r}(t)$, $E_{r^{\prime}}(t)$, respectively. By the convexity of $g\left(r_{1}, r_{2}\right)$, we have

$$
g\left(\lambda r_{1}+\bar{\lambda} r_{1}^{\prime}, \lambda r_{2}+\bar{\lambda} r_{2}^{\prime}\right) \leq \lambda g\left(r_{1}, r_{2}\right)+\bar{\lambda} g\left(r_{1}^{\prime}, r_{2}^{\prime}\right)
$$

Hence, the energy removal curve of $\left(\lambda r_{1 i}+\bar{\lambda} r_{1 i}^{\prime}, \lambda r_{2 i}+\bar{\lambda} r_{2 i}^{\prime}\right)$, denoted as $E_{\bar{r}}(t)$, satisfies

$$
\begin{aligned}
E_{\bar{r}}(t) & \leq \max \left\{E_{r}(t), E_{r^{\prime}}(t)\right\} \quad \forall t \in[0, T] \\
& \leq \sum_{i=0}^{h(t)} E_{i}, \quad \forall t \in[0, T]
\end{aligned}
$$

Hence, $\left(\lambda r_{1 i}+\bar{\lambda} r_{1 i}^{\prime}, \lambda r_{2 i}+\bar{\lambda} r_{2 i}^{\prime}\right)$ is also in $\mathcal{P}_{R}(T)$.

Extending the constraint set of problem (24) to $\mathcal{P}_{R}(T)$ does not change the optimum value of the objective, because energy overflows are sub-optimal. As $\mathcal{P}_{R}(T)$ is a convex set and the objective function is concave, there exists a unique optimum solution for the extended problem, and this is within the feasible set of the original problem. Hence, we conclude that there exists a unique global optimum solution for (24).

We write the Lagrangian for the optimization problem in (24) as:

$$
\begin{aligned}
\mathcal{L}= & \mu_{1} \sum_{i=1}^{K+1} r_{1 i} \ell_{i}+\mu_{2} \sum_{i=1}^{K+1} r_{2 i} \ell_{i} \\
& -\sum_{k=1}^{K+1} \lambda_{k}\left(\sum_{i=1}^{k} g\left(r_{1 i}, r_{2 i}\right) \ell_{i}-\sum_{i=0}^{k-1} E_{i}\right) \\
& -\sum_{k=1}^{K} \eta_{k}\left(\sum_{i=0}^{k} E_{i}-\sum_{i=1}^{k} g\left(r_{1 i}, r_{2 i}\right) \ell_{i}-E_{\text {max }}\right) \\
& +\sum_{i=1}^{K+1} \gamma_{1 i} r_{1 i}+\sum_{i=1}^{K+1} \gamma_{2 i} r_{2 i}
\end{aligned}
$$

Taking the derivatives of $\mathcal{L}$ in (29) with respect to $r_{1 i}$ and $r_{2 i}$, and setting them to zero, we have, for all $i$

$$
\begin{aligned}
& \mu_{1}+\gamma_{1 i}=\left(\sum_{k=i}^{K+1} \lambda_{k}-\sum_{k=i}^{K} \eta_{k}\right) 2^{2\left(r_{1 i}+r_{2 i}\right)} \\
& \mu_{2}+\gamma_{2 i} \\
& =\left(\sum_{k=i}^{K+1} \lambda_{k}-\sum_{k=i}^{K} \eta_{k}\right)\left(2^{2\left(r_{1 i}+r_{2 i}\right)}+\left(\sigma^{2}-1\right) 2^{2 r_{2 i}}\right)
\end{aligned}
$$

Additional complimentary slackness conditions are

$$
\begin{aligned}
\gamma_{1 k} r_{1 k}=0, \quad \gamma_{2 k} r_{2 k} & =0 \\
\lambda_{k}\left(\sum_{i=1}^{k} g\left(r_{1 i}, r_{2 i}\right) \ell_{i}-\sum_{i=0}^{k-1} E_{i}\right) & =0 \\
\eta_{k}\left(\sum_{i=0}^{k} E_{i}-\sum_{i=1}^{k} g\left(r_{1 i}, r_{2 i}\right) \ell_{i}-E_{\max }\right) & =0
\end{aligned}
$$

The optimal policy is the solution of (30) and (31) with the complimentary slackness conditions in (32)-(34). In the next lemma, we prove a key structural property of the optimal policy.

Lemma 3 Optimal total transmit power of the transmitter is independent of the values of $\mu_{1}, \mu_{2}$, and, in particular, it is the same as the single-user optimal transmit power, i.e., for $\mu_{2}=0$.

Proof: From the rate-power relation in (6) and the KKT optimality conditions in (30) and (31), we have

$$
\begin{aligned}
g\left(r_{1 i}, r_{2 i}\right) & =\frac{\mu_{2}+\gamma_{2 i}}{\left(\sum_{k=i}^{K+1} \lambda_{k}-\sum_{k=i}^{K} \eta_{k}\right)}-\sigma^{2} \\
& \geq 2^{2\left(r_{1 i}+r_{2 i}\right)}-1 \\
& =\frac{\mu_{1}+\gamma_{1 i}}{\left(\sum_{k=i}^{K+1} \lambda_{k}-\sum_{k=i}^{K} \eta_{k}\right)}-1 \\
& \geq \frac{\mu_{1}}{\left(\sum_{k=i}^{K+1} \lambda_{k}-\sum_{k=i}^{K} \eta_{k}\right)}-1
\end{aligned}
$$

Here, (36) becomes an equality when $r_{2 i}=0$. Therefore, by (35)-(38), whenever $r_{2 i}>0$,

$$
\begin{aligned}
P_{i} & =\frac{\mu_{2}}{\left(\sum_{k=i}^{K+1} \lambda_{k}-\sum_{k=i}^{K} \eta_{k}\right)}-\sigma^{2} \\
& >\frac{\mu_{1}}{\left(\sum_{k=i}^{K+1} \lambda_{k}-\sum_{k=i}^{K} \eta_{k}\right)}-1
\end{aligned}
$$

where $P_{i}=g\left(r_{1 i}, r_{2 i}\right)$ is the total power in the $i$ th epoch. On the other hand, if $r_{2 i}=0$, we have

$$
\begin{aligned}
P_{i} & =\frac{\mu_{1}}{\left(\sum_{k=i}^{K+1} \lambda_{k}-\sum_{k=i}^{K} \eta_{k}\right)}-1 \\
& >\frac{\mu_{2}}{\left(\sum_{k=i}^{K+1} \lambda_{k}-\sum_{k=i}^{K} \eta_{k}\right)}-\sigma^{2}
\end{aligned}
$$

Hence, we have

$$
\begin{aligned}
P_{i}=\max \left\{\frac{\mu_{1}}{\left(\sum_{k=i}^{K+1} \lambda_{k}-\sum_{k=i}^{K} \eta_{k}\right)}-1,\right. \\
\\
\left(\frac{\mu_{2}}{\left(\sum_{k=i}^{K+1} \lambda_{k}-\sum_{k=i}^{K} \eta_{k}\right)}-\sigma^{2}\right\}
\end{aligned}
$$

The Lagrange multipliers $\lambda_{i}, \eta_{i}$ and $\gamma_{i}$ are uniquely determined by the complimentary slackness conditions in (32)-(34) as well as the equality condition, $\sum_{i=1}^{K+1} P_{i} \ell_{i}=\sum_{i=1}^{K+1} E_{i}$. The last equality is needed as otherwise unused energy could yield higher performance. Therefore, the optimum power allocation is unique. We will next argue that the optimum power allocation does not depend on $\mu_{1}, \mu_{2}$.

We know that $E_{i} \leq E_{\max }$ for all $i$. We will first consider the case $E_{i} \neq E_{\max }$. In this case, if $\lambda_{i}>0$ then $\eta_{i}=0$ and if $\eta_{i}>0, \lambda_{i}=0$. This is due to the fact that the constraints in (16) and (17) cannot be simultaneously satisfied 
with equality unless $E_{i}=E_{\max }$. Hence, total power $P_{i}$ is monotone increasing in each interval $\left[s_{i_{1}}, s_{i_{2}}\right)$ where $E_{\max }$ constraint is consecutively satisfied in the $i_{1}$ th and $i_{2}$ th epochs, i.e., $\eta_{i_{1}}, \eta_{i_{2}}>0$ and $\eta_{i}=0$ for $i_{1}<i<i_{2}$. Whenever an energy causality constraint is satisfied with equality, say in epoch $i, \lambda_{i}>0$; this leads to a strict increase in $P_{i}$ in view of (43). Thus, equality of energy causality constraints leads to an increase while that of $E_{\max }$ constraint leads to a decrease in the total power. Imposing the energy constraint at time $T$ as an equality, we get exactly the same power policy as the optimal power policy in the single user $E_{\max }$ constrained average throughput maximization problem in [3], i.e, in the special case of $\mu_{2}=0$. Moreover, this characterization is the same for any $\mu_{1}, \mu_{2} \geq 0$.

If $E_{i}=E_{\max }$ for some epoch $i$, in order to accommodate the future energy arrivals, the energy arrived should be equal to the energy consumed in $\left[0, s_{i}\right)$. This way, the problem can be separately solved in two intervals $\left[0, s_{i}\right)$ and $\left[s_{i}, T\right]$. In general, if $E_{i}=E_{\max }$ at more than one epoch, we can divide the problem into that many subproblems and solve them separately by allowing energy arrived equal the energy consumed in the corresponding intervals.

Therefore, irrespective of the values of $\mu_{1}, \mu_{2}$, the unique total power allocation can be found by the directional waterfilling algorithm introduced in [3]. The algorithm is based on a water-energy analogy. Each arriving energy is first allocated to the corresponding epoch, and if the power level in an epoch is higher than the one in the next epoch, then some energy is transferred from the past to the future to equalize the power levels. However, no energy can be transferred from right to left (i.e., from the future to the past) due to energy causality. Direction selectivity of the algorithm is indicated by right permeable taps. Moreover, $E_{\max }$ constrains the amount of energy that can be transferred from the past to the future. If the equalizing water level requires more than $E_{\text {max }}-E_{i}$ amount of energy to be transferred to the $i+1$ st epoch, then only $E_{\max }-E_{i}$ can be transferred.

After finding the total power allocation using directional water-filling, the optimization problem in (24) can be separately solved over each duration $\left[s_{i-1}, s_{i}\right)$. Specifically, for a given sequence of total transmit powers $P_{i}, i=1, \ldots, K$, the local optimization problem in epoch $i$ becomes

$$
\begin{aligned}
\max _{r_{1 i}, r_{2 i}} & \mu_{1} r_{1 i}+\mu_{2} r_{2 i} \\
\text { s.t. } & g\left(r_{1 i}, r_{2 i}\right) \leq P_{i}
\end{aligned}
$$

As in [1], we define a constant power level $P_{c}$ as

$$
P_{c}=\left(\frac{\mu_{1}\left(\sigma^{2}-1\right)}{\mu_{2}-\mu_{1}}-1\right)^{+}
$$

This constant value represents the cut-off power level for the stronger user in the optimal policy.

Lemma 4 A point $\left(B_{1}, B_{2}\right)$ on the boundary of $\mathcal{D}(T)$, which is equally represented by $\left(\mu_{1}, \mu_{2}\right)$, is achieved by the following policy: If in an epoch the total transmit power level is below
$P_{c}$ in (45), then, only the stronger user's data is transmitted; otherwise, both users' data are transmitted and the stronger user's power share is $P_{c}$.

Proof: Since the total power level consumed in epoch $i$, $P_{i}$, is obtained directly irrespective of the values of $\mu_{1}$ and $\mu_{2}$, the optimization problem in (44) is performed separately over each duration $\left[s_{i-1}, s_{i}\right)$. Note that we relax the power constraint in (44) as an inequality so that it can be stated as a convex optimization problem. It is clear that we do not lose optimality by doing so as the objective function is monotone increasing with respect to total power.

After necessary algebraic steps, one can show as in [1] that if $\frac{\mu_{2}}{\mu_{1}}<\frac{1+P_{i}}{\sigma^{2}+P_{i}}$, then the optimizing values of the variables, $r_{1 i}^{*}$ and $r_{2 i}^{*}$ are

$$
\begin{aligned}
r_{1 i}^{*} & =\frac{1}{2} \log \left(1+P_{i}\right) \\
r_{2 i}^{*} & =0
\end{aligned}
$$

If $\frac{1+P_{i}}{\sigma^{2}+P_{i}} \leq \frac{\mu_{2}}{\mu_{1}} \leq \sigma^{2}$, then

$$
\begin{aligned}
& r_{1 i}^{*}=\frac{1}{2} \log \left(\frac{\mu_{1}\left(\sigma^{2}-1\right)}{\mu_{2}-\mu_{1}}\right) \\
& r_{2 i}^{*}=\frac{1}{2} \log \left(\frac{\left(\mu_{2}-\mu_{1}\right)\left(P_{i}+\sigma^{2}\right)}{\mu_{2}\left(\sigma^{2}-1\right)}\right)
\end{aligned}
$$

Finally, if $\frac{\mu_{2}}{\mu_{1}}>\sigma^{2}$, then

$$
\begin{aligned}
& r_{1 i}^{*}=0 \\
& r_{2 i}^{*}=\frac{1}{2} \log \left(1+\frac{P_{i}}{\sigma^{2}}\right)
\end{aligned}
$$

The statement of the lemma is just a compact expression of the above optimality conditions in (46)-(51).

Based on Lemmas 3 and 4, for fixed $T$, we observe that the optimal policies that achieve the boundary of $\mathcal{D}(T)$ have a common total power and its splitting between the two users depends on $\mu_{1}, \mu_{2}$. Therefore, for different values of $\mu_{2} / \mu_{1}$, the optimal policy achieves different boundary points on the maximum departure region. Varying the value of $\mu_{2} / \mu_{1}$ traces the boundary of $\mathcal{D}(T)$.

\section{Minimum Transmission Completion Time For Given $\left(B_{1}, B_{2}\right)$}

In this section, we consider the transmission completion time minimization problem for a given fixed $\left(B_{1}, B_{2}\right)$. The optimization problem can be formulated as:

$$
\begin{array}{rl}
\min _{\mathbf{r}_{1}, \mathbf{r}_{2}} & T \\
\text { s.t. } & \sum_{i=1}^{k} g\left(r_{1 i}, r_{2 i}\right) \ell_{i} \leq \sum_{i=1}^{k-1} E_{i}, \quad 1 \leq k \leq K+1 \\
& \sum_{i=0}^{k} E_{i}-\sum_{i=1}^{k} g\left(r_{1 i}, r_{2 i}\right) \ell_{i} \leq E_{\max }, \quad 1 \leq k \leq K \\
& \sum_{i=1}^{K+1} r_{1 i} \ell_{i}=B_{1}, \quad \sum_{i=1}^{K+1} r_{1 i} \ell_{i}=B_{2}
\end{array}
$$


where $K=K(T)$ is the number of energy arrivals over $[0, T)$, and $l_{K(T)+1}=T-s_{K(T)}$. Since $K(T)$ depends on $T$, the optimization problem in (52) is not convex in general.

We observe that (52) is the "dual" problem of finding the departure region for fixed $T$ in (24) in the sense that, if the minimum transmission completion time for $\left(B_{1}, B_{2}\right)$ is $T$, then $\left(B_{1}, B_{2}\right)$ must lie on the boundary of $\mathcal{D}(T)$. We also note that in the optimal policy for $E_{\max }=\infty$ [1], both receivers' transmissions end at the same time. However, transmissions do not necessarily end at the same time when there is an additional $E_{\max }$ constraint. Since the total power level is not monotonically increasing in the $E_{\max }$ constrained case, the cut-off power level $P_{c}$ may be higher than the total power allocated in the final epoch rendering only the stronger user transmit in the final epoch.

\section{A. Algorithm to Find the Optimal Policy Given $\left(B_{1}, B_{2}\right)$}

$\left(B_{1}, B_{2}\right)$ must lie on the boundary of $\mathcal{D}\left(T_{\min }\right)$. Hence, without losing optimality we restrict our attention to the policies which allocate the total power by directional waterfilling and have the cut-off power structure. As the initial step, we suppose that the transmitter transmits only to the stronger user with an arbitrary $P_{c}$ and find the transmission completion time for the stronger user

$$
T_{1}=\frac{B_{1}}{f\left(P_{c}\right)}
$$

For this fixed $T_{1}$, we run the directional water-filling algorithm and find the total power allocation $P_{1}, P_{2}, \ldots, P_{K\left(T_{1}\right)+1}$ with the deadline $T_{1}$. The number of bits transmitted to the stronger user is

$$
D_{1}\left(T_{1}, P_{c}\right)=\sum_{i=1}^{K\left(T_{1}\right)+1} \frac{1}{2} \log \left(1+P_{i}-\left[P_{i}-P_{c}\right]^{+}\right) \ell_{i}
$$

We allocate the remaining power $\left[P_{i}-P_{c}\right]^{+}$to the weaker user and calculate the total bits departed from the weaker user's queue by deadline $T_{1}$ as

$$
D_{2}\left(T_{1}, P_{c}\right)=\sum_{i=1}^{K\left(T_{1}\right)+1} \frac{1}{2} \log \left(1+\frac{\left[P_{i}-P_{c}\right]^{+}}{P_{c}+\sigma^{2}}\right) \ell_{i}
$$

$D_{2}\left(T_{1}, P_{c}\right)$ is monotonically decreasing with $P_{c}$ for fixed $T_{1}$. In fact, $D_{2}\left(T_{1}, P_{c}\right)$ takes its maximum value at $P_{c}=0$ and as $P_{c}$ is increased, the achievable bit departure pairs travel on the boundary of $\mathcal{D}\left(T_{1}\right)$ from one extreme to the other.

We divide the bit departure plane into 5 regions as shown in Fig. 7. The regions are bordered by the constant $B_{1}, B_{2}$ lines and the $\mathcal{D}\left(T_{\text {min }}\right)$ curve. Region (1) is $D_{1} \leq B_{1}$ and $D_{2} \leq B_{2}$. Regions (2) and (3) combined represent the northwest part, i.e., $D_{1} \leq B_{1}$ and $D_{2} \geq B_{2}$. The border between regions 2 and 3 is the $\mathcal{D}\left(T_{\text {min }}\right)$ curve. Region (5) is bordered by the constant $B_{1}$ line and the $\mathcal{D}\left(T_{\min }\right)$ curve. The rest of the first quadrant is region (4). We start the problem with the knowledge of $\left(B_{1}, B_{2}\right)$. While we know that $\left(B_{1}, B_{2}\right)$ must lie on the boundary of $\mathcal{D}\left(T_{\text {min }}\right)$, we do not know $\mathcal{D}\left(T_{\text {min }}\right)$ or $T_{\min }$. We want to find $T_{\min }$ and the policy that achieves it.
After the initial step, we have $D_{1}\left(T_{1}, P_{c}\right) \leq B_{1}$ since $P_{i}<$ $P_{c}$ may occur in some epochs. Hence, the initial operating point lies in one of regions (1), (2), (3).

If the operating point lies in the interior of region (1), it implies that $\left(B_{1}, B_{2}\right)$ transmission cannot be completed by $T_{1}$. Therefore, we decrease $P_{c}$, obtain another $T_{1}$ according to (53), and repeat the procedure, until we leave this region.

If by performing the initialization or the previous step, the operating point hits the $B_{1}$ line, i.e., $D_{1}\left(\frac{B_{1}}{f\left(P_{c}\right)}, P_{c}\right)=B_{1}$, while $D_{2}\left(\frac{B_{1}}{f\left(P_{c}\right)}, P_{c}\right)<B_{2}$, as shown in Fig. 7(a), this implies that $P_{c}<P_{i}$ for all epochs $i$ and even if we further decrease $P_{c}$ to increase $D_{2}$, we always have $D_{1}\left(\frac{B_{1}}{f\left(P_{c}\right)}, P_{c}\right)=$ $B_{1}$. Hence, similar to the algorithm for the unlimited battery case in [1], we apply bisection only on $P_{c}$ and approach $D_{2}\left(\frac{B_{1}}{f\left(P_{c}\right)}, P_{c}\right)=B_{2}$ sufficiently. For the final value of $P_{c}$, $T_{\text {min }}=\frac{B_{1}}{f\left(P_{c}\right)}$.

Then, we consider the scenario when the operating point enters into region (2) or (3), i.e., $D_{2}\left(\frac{B_{1}}{f\left(P_{c}\right)}, P_{c}\right)>B_{2}$ while $D_{1}\left(\frac{B_{1}}{f\left(P_{c}\right)}, P_{c}\right) \leq B_{1}$. For this scenario, we fix $T_{1}$ and increase $P_{c}$ such that $D_{2}\left(T_{1}, P_{c}\right)=B_{2}$. This brings us to the horizontal $B_{2}$ line, as shown in Fig. 7(b). Depending on the updated $D_{1}$ under this policy, the operating point lies either on the left or on the right of the $\left(B_{1}, B_{2}\right)$ point.

If we end up at $D_{1}\left(T_{1}, P_{c}\right)<B_{1}$, it implies $T_{1}<T_{\text {min }}$. Then, we decrease $P_{c}$, and obtain a larger $T_{1}$ from (53). Another round of directional water-filing results $D_{2}>B_{2}$, and brings the operating point back into region (2) and (3).

If we end up at $D_{1}\left(T_{1}, P_{c}\right)>B_{1}$, it implies $T_{1}>T_{m i n}$. then we fix $P_{c}$ and decrease $T_{1}$ only. By doing this, we decrease $D_{1}$ and $D_{2}$ at the same time. This takes the operating point into region (5) or (1) or it remains in region (4). Again, we fix $T_{1}$, increase $P_{c}$, and bring the operating point back to the horizontal $B_{2}$ line. This brings us back to one of the previously considered cases depending on whether $D_{1}\left(T_{1}, P_{c}\right)$ is greater or smaller than $B_{1}$.

For all of the above cases, we carefully control the step size when we do the adjustment of $P_{c}$ and $T_{1}$, to make sure that the operating point gets closer to the $\left(B_{1}, B_{2}\right)$ at each step. Existences of such step sizes are due to continuity of $D_{1}\left(T_{1}, P_{c}\right)$ and $D_{2}\left(T_{1}, P_{c}\right)$.

\section{A Numerical Example}

We consider a classical band-limited AWGN broadcast channel with $W=1 \mathrm{MHz}$ and noise power spectral density $N_{0}=10^{-19} \mathrm{~W} / \mathrm{Hz}$. The path loss between the transmitter and the strong user is $100 \mathrm{~dB}$ and between the transmitter and the weak user is $105 \mathrm{~dB}$. We have

$$
\begin{aligned}
r_{1} & =W \log _{2}\left(1+\frac{\alpha P h_{1} 10^{-3}}{N_{0} W}\right) \\
& =\log _{2}(1+\alpha P) \mathrm{Mbps}
\end{aligned}
$$



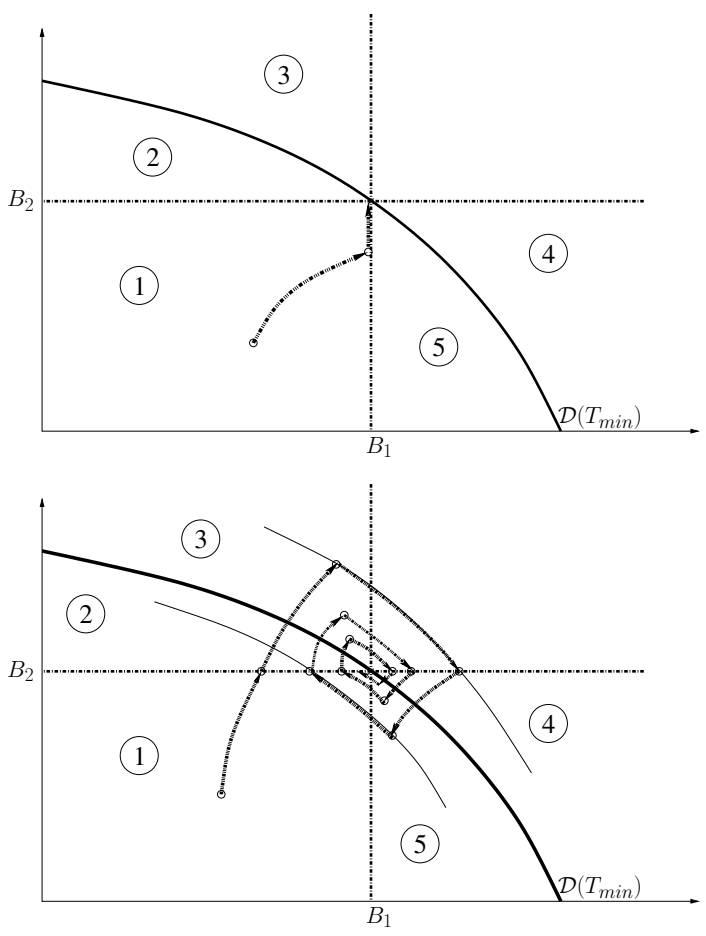

Fig. 7. (a) If the algorithm starts in region (1) and hits $D_{1}\left(T_{1}, P_{c}\right)=$ $B_{1}$, then the trajectory does not deviate from the constant $B_{1}$ line. (b) If $D_{1}\left(T_{1}, P_{c}\right)<B_{1}$ and $D_{2}\left(T_{1}, P_{c}\right)=B_{2}$ is achieved, then a bisection algorithm converges to the desired $\left(B_{1}, B_{2}\right)$ point yielding the minimum $T$.

and

$$
\begin{aligned}
r_{2} & =W \log _{2}\left(1+\frac{(1-\alpha) P h_{2} 10^{-3}}{\alpha P 10^{-3} h_{2}+N_{0} W}\right) \\
& =\log _{2}\left(1+\frac{(1-\alpha) P}{\alpha P+10^{0.5}}\right) \mathrm{Mbps}
\end{aligned}
$$

We assume $E_{\max }=10 \mathrm{~mJ}$ and the energy arrivals occur at times $[2,5,8,9,12]$ seconds with amounts $[3,6,9,8,9] \mathrm{mJ}$. Initial energy in the battery is $E_{0}=8 \mathrm{~mJ}$. The number of bits to be served for the strong and weak users are $B_{1}=22 \mathrm{Mbits}$ and $B_{2}=3$ Mbits, respectively.

Our algorithm yields the optimal transmission policy, which is shown in Fig. 8. Initial energy in the battery and the first two energy arrivals are spread till $t=8 \mathrm{~s}$. However, only $2 \mathrm{~mJ}$ energy can flow from the time interval $[8,9]$ to $[9,12]$ as $E_{\max }=10 \mathrm{~mJ}$ constrains the energy flow. This, in turn, breaks the monotonicity in the total transmit power. In the optimal policy, $P_{c}=2.15 \mathrm{~mW}$ is found, while in the first three epochs the transmit power is allocated as $2.125 \mathrm{~mW}$. Therefore, only the stronger user's data is transmitted in the first three epochs. In the remaining epochs, both users' data are transmitted simultaneously with transmit power $P_{4}=7$ $\mathrm{mW}$ in $[8,9] \mathrm{s}, P_{5}=3.33 \mathrm{~mW}$ in $[9,12] \mathrm{s}$ and $P_{6}=6.66$ $\mathrm{mW}$ in $[12,13.5] \mathrm{s}$.

\section{CONCLUSION}

In this paper, we considered the transmission completion time minimization problem in a two-user broadcast channel

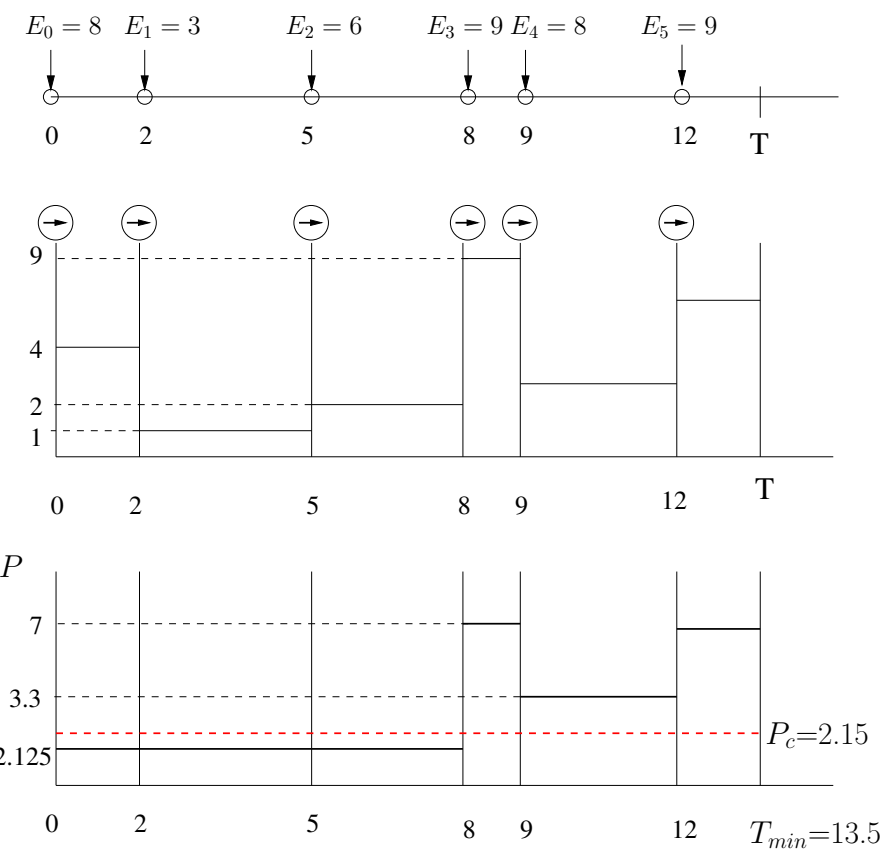

Fig. 8. (a) Energy arrivals occur at $[2,5,8,9,12]$ seconds with amounts $[3,6,9,8,9] \mathrm{mJ}$ with initial energy in the battery at time zero $E_{0}=8 \mathrm{~mJ}$. (b) Directional water-filling with right permeable taps. (c) The resulting optimal transmission policy.

where the transmitter harvests energy from nature and saves it in a battery of finite storage capacity. We characterized the structural properties of the optimal policy by means of the dual problem of maximizing the weighted sum of bits served for each user by a fixed deadline. We found that the total power allocation is the same as the single-user power allocation, which is found by a directional water-filling algorithm. Moreover, there exists a cut-off power level such that only the total power above this level is assigned to the weaker user. This structure enabled us to develop an optimal offline algorithm which uses single-user directional waterfilling repetitively.

\section{REFERENCES}

[1] J. Yang, O. Ozel, and S. Ulukus, "Broadcasting with an energy harvesting rechargeable transmitter," IEEE Trans. on Wireless Communications, submitted, October 2010. Also available at [arXiv:1010.2993].

[2] K. Tutuncuoglu and A. Yener, "Optimum transmission policies for battery limited energy harvesting systems," IEEE Trans. on Wireless Communications, submitted, September 2010. Also available at [arXiv:1010.6280].

[3] O. Ozel, K. Tutuncuoglu, J. Yang, S. Ulukus, and A. Yener, "Transmission with energy harvesting nodes in fading wireless channels: Optimal policies," IEEE Journal on Selected Areas in Communications, submitted, October 2010.

[4] J. Yang and S. Ulukus, "Transmission completion time minimization in an energy harvesting system," in CISS, March 2010.

[5] J. Yang and S. Ulukus, "Optimal packet scheduling in an energy harvesting communication system," IEEE Trans. on Communications, submitted, June 2010. Also available at [arXiv:1010.1295].

[6] M. A. Antepli, E. Uysal-Biyikoglu, and H. Erkal, "Optimal packet scheduling on an energy harvesting broadcast link," IEEE Journal on Selected Areas in Communications, submitted, October 2010. Also available at [arXiv: 1010.4876].

[7] T. M. Cover and J. Thomas, Elements of Information Theory. John Wiley and Sons Inc., 2006. 